\title{
Left-right supersymmetric option at a high-energy upgrade of the LHC
}

\author{
Mariana Frank $\odot,{ }^{1, *}$ Benjamin Fuks $\odot,{ }^{2, \dagger}$ Katri Huitu, ${ }^{3, \ddagger}$ Subhadeep Mondal $\odot,{ }^{3, \S}$ Santosh Kumar Rai, ${ }^{4, \|}$ and Harri Waltari $\odot^{5, \text {, }}$ \\ ${ }^{1}$ Department of Physics, Concordia University, 7141 Sherbrooke Street West, Montreal, \\ QC, Canada H4B IR6 \\ ${ }^{2}$ Sorbonne Université, CNRS, Laboratoire de Physique Théorique et Hautes Énergies, LPTHE, F-75005 \\ Paris, France and Institut Universitaire de France, 103 boulevard Saint-Michel, 75005 Paris, France \\ ${ }^{3}$ Department of Physics, and Helsinki Institute of Physics, University of Helsinki, \\ P. O. Box 64, FI-00014 Helsinki, Finland \\ ${ }^{4}$ Regional Centre for Accelerator-based Particle Physics, Harish-Chandra Research Institute, HBNI, \\ Chhatnag Road, Jhusi, Prayagraj 211019, India \\ ${ }^{5}$ Department of Physics and Astronomy, University of Southampton, Highfield, Southampton SO17 1BJ, \\ United Kingdom and Department of Particle Physics, Rutherford Appleton Laboratory, \\ Didcot OX11 OQX, United Kingdom
}

(Received 23 March 2020; accepted 18 May 2020; published 12 June 2020)

\begin{abstract}
We investigate the possibility that a minimal realization of left-right supersymmetry can be reachable at a high-energy upgrade of the LHC, expected to operate at a center-of-mass energy of $27 \mathrm{TeV}$. This minimal scenario has a relatively light $S U(2)_{R}$ doubly charged Higgs boson, which could decay dominantly into tau-lepton pairs. We explore the associated signals comprised of at least three hadronically decaying taus, or with at least two hadronic taus and one same-sign-same-flavor charged lepton pair. Our analysis shows that the former signature is challenging to use for getting handles on the signal due to the large corresponding background, and that the latter one can lead to a handful of new physics events in an almost backgroundfree environment. We however find that a signal comprised of three hadronically decaying tau leptons is likely to be observed at a low luminosity of proton-proton collisions at a $27 \mathrm{TeV}$ upgrade of the LHC.
\end{abstract}

DOI: 10.1103/PhysRevD.101.115014

\section{INTRODUCTION}

During the last years of operation at the Large Hadron Collider (LHC), no significant deviation from the Standard Model (SM) predictions has been found. Still, the SM as it stands is incomplete as it fails to explain, for instance, neutrino masses, dark matter and the baryon-antibaryon asymmetry of the Universe. However, new particles and interactions have failed (so far) to materialize at the LHC. Of all the candidates for physics beyond the SM, weak scale supersymmetry [1,2] is amongst the most promising ones. It associates one partner of opposite statistics with each of the SM degrees of freedom and unifies the Poincaré

\footnotetext{
*mariana.frank@concordia.ca

fuks@lpthe.jussieu.fr

*katri.huitu@helsinki.fi

\$subhadeep.mondal@helsinki.fi

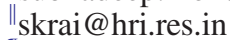

"h.waltari@soton.ac.uk
}

Published by the American Physical Society under the terms of the Creative Commons Attribution 4.0 International license. Further distribution of this work must maintain attribution to the author(s) and the published article's title, journal citation, and DOI. Funded by SCOAP ${ }^{3}$. symmetry with the internal gauge symmetries. This setup leads to an elegant solution to some of the limitations of the $\mathrm{SM}$, as for instance concrete and realistic supersymmetric realizations usually include a natural dark matter candidate as the lightest supersymmetric particle (LSP).

On the other hand, left-right symmetric models [3-5] provide a natural mechanism to generate neutrino masses. In this case, parity is a symmetry of the theory (that has to be further broken dynamically) and the strong $C P$ problem is solved, thanks to the enlarged gauge symmetry group [6-8]. In the supersymmetric context, in which both supersymmetry and left-right motivations are combined, the same extended gauge symmetry reason leads additionally to the automatic absence of any $R$-parity-violating interaction. This therefore prevents the proton from being unstable and guarantees a viable dark matter candidate as the LSP. However, the simplest left-right supersymmetric realizations often predict upper bounds for particle masses that do not easily agree with the latest non-observations of any hint for new physics in LHC data, at least when treelevel calculations are in order. Already the first proposal for a left-right supersymmetric (LRSUSY) model hence suggested an $S U(2)_{R}$ charged gauge boson with a mass satisfying $m_{W_{R}} \lesssim 1 \mathrm{TeV}$ [9], which is today largely ruled 
out by the results of the LHC experiments [10,11]. This has consequently led to the development of LRSUSY models featuring an extended Higgs sector, so that the $S U(2)_{R}$ boson masses could be pushed to a higher scale [12].

In this case, left-right symmetry breaking is often minimally built through $S U(2)_{R}$ scalar triplets featuring two neutral, one singly charged and one doubly charged Higgs degrees of freedom. At tree-level, the latter generally acquires a vacuum expectation value (VEV) at the global minimum of the potential that corresponds to a configuration that is lower in energy than the one in which only the neutral states develop a VEV. This problem can be cured by invoking large contributions either from nonrenormalizable operators $[6,8,13]$ or through loop corrections [14-16], or by spontaneous $R$-parity breaking [17]. The most appealing option consists of the second one, in which loop corrections stabilize the charge-conserving vacuum [16]. In this setup, the model turns out to be quite predictable, at least for what concern the properties of the $W_{R}$ boson (and in particular its mass).

On different grounds, imposing a dark matter candidate with the right features, that for instance leads to a relic density in agreement with Planck data, further restricts the possibilities for the particle spectrum as the LSP has to lie within some mass range below $1 \mathrm{TeV}[18,19]$. With this phenomenologically constrained version of LRSUSY at hand, we investigate in this work whether the future highluminosity phase of the LHC (HL-LHC) or its proposed $27 \mathrm{TeV}$ energy upgrade, the so-called high-energy LHC (HE-LHC) [20], could observe or rule out the model once and for all.

The rest of this work is organized as follows. In Sec. II, we briefly describe our theoretical framework, detailing in particular how a LRSUSY discovery at the HL-LHC could not happen. In Sec. III, we focus on this most pessimistic case and design a set of representative benchmark scenarios. We then demonstrate how the HE-LHC could provide handles on the model. We summarize and conclude in Sec. IV.

\section{LEFT-RIGHT SUPERSYMMETRY}

Left-right symmetric models [3-5] are based on the gauge group $S U(3)_{C} \times S U(2)_{L} \times S U(2)_{R} \times U(1)_{B-L}$. The matter sector is defined by three families of left- and right-handed quark and lepton supermultiplets,

$$
\begin{gathered}
Q_{L}=\left(\begin{array}{c}
u_{L} \\
d_{L}
\end{array}\right) \sim(\mathbf{3}, \mathbf{2}, \mathbf{1})_{\frac{1}{3}}, \quad Q_{R}=\left(\begin{array}{c}
d_{R}^{c} \\
-u_{R}^{c}
\end{array}\right) \sim\left(\overline{\mathbf{3}}, \mathbf{1}, \mathbf{2}^{*}\right)_{-\frac{1}{3}}, \\
L_{L}=\left(\begin{array}{c}
\nu_{L} \\
e_{L}
\end{array}\right) \sim(\mathbf{1}, \mathbf{2}, \mathbf{1})_{-1}, \quad L_{R}=\left(\begin{array}{c}
e_{R}^{c} \\
-N^{c}
\end{array}\right) \sim\left(\mathbf{1}, \mathbf{1}, \mathbf{2}^{*}\right)_{1},
\end{gathered}
$$

where we include in our notation the representations of the various fields under the LRSUSY gauge group, with the $U(1)_{B-L}$ charge given as a subscript. Compared with the usual minimal supersymmetric standard model (MSSM), the spectrum features a right-handed neutrino field $N$ (as part of the $S U(2)_{R}$ lepton doublet $L_{R}$ ), so that Dirac neutrino mass term are allowed in the superpotential. The Higgs sector of the model is quite rich and includes an $S U(2)_{R}$ Higgs triplet to break the $S U(2)_{R} \times U(1)_{B-L}$ symmetry, its $S U(2)_{L}$ counterpart to preserve left-right parity, as well as a pair of $S U(2)_{L} \times S U(2)_{R}$ Higgs bidoublets that are required to generate all SM fermion masses. Neutrino masses are hence generated through a combination of the Type-II [21-26] and Type I [25-32] seesaw mechanisms, after the breaking of the left-right symmetry. The Higgs sector moreover includes an extra gauge singlet that allows for the shift of the left-right symmetry breaking scale well beyond the $\mathrm{TeV}$ regime. The Higgs superfield content and the associated representations under the LRSUSY gauge group are hence summarized as

$$
\begin{aligned}
\Phi_{a} & =\left(\begin{array}{cc}
\Phi_{a 1}^{+} & \Phi_{a 1}^{0} \\
\Phi_{a 2}^{0} & \Phi_{a 2}^{-}
\end{array}\right) \sim\left(\mathbf{1}, \mathbf{2}, \mathbf{2}^{*}\right)_{0}, \\
\Delta_{L} & =\left(\begin{array}{cc}
\frac{1}{\sqrt{2}} \Delta_{L}^{-} & \Delta_{L}^{0} \\
\Delta_{L}^{--} & -\frac{1}{\sqrt{2}} \Delta_{L}^{-}
\end{array}\right) \sim(\mathbf{1}, \mathbf{3}, \mathbf{1})_{-2}, \\
\delta_{L} & =\left(\begin{array}{cc}
\frac{1}{\sqrt{2}} \delta_{L}^{+} & \delta_{L}^{++} \\
\delta_{L}^{0} & -\frac{1}{\sqrt{2}} \delta_{L}^{+}
\end{array}\right) \sim(\mathbf{1}, \mathbf{3}, \mathbf{1})_{2}, \\
\Delta_{R} & =\left(\begin{array}{cc}
\frac{1}{\sqrt{2}} \Delta_{R}^{-} & \Delta_{R}^{0} \\
\Delta_{R}^{--} & -\frac{1}{\sqrt{2}} \Delta_{R}^{-}
\end{array}\right) \sim(\mathbf{1}, \mathbf{1}, \mathbf{3})_{-2}, \\
\delta_{R} & =\left(\begin{array}{cc}
\frac{1}{\sqrt{2}} \delta_{R}^{+} & \delta_{R}^{++} \\
\delta_{R}^{0} & -\frac{1}{\sqrt{2}} \delta_{R}^{+}
\end{array}\right) \sim(\mathbf{1}, \mathbf{1}, \mathbf{3})_{2}, \\
S & \sim(\mathbf{1}, \mathbf{1}, \mathbf{1})_{0} .
\end{aligned}
$$

The model Lagrangian includes, on top of usual gaugeinvariant kinetic terms for all fields, supersymmetric interaction terms originating from the superpotential $W$ and a soft supersymmetry-breaking Lagrangian. The superpotential reads

$$
\begin{aligned}
W= & Q_{L}^{T} \mathbf{Y}_{Q}^{(i)} \Phi_{i} Q_{R}+L_{L}^{T} \mathbf{Y}_{L}^{(i)} \Phi_{i} L_{R}+L_{L}^{T} \mathbf{h}_{L L} \delta_{L} L_{L} \\
& +L_{R}^{T} \mathbf{h}_{R R} \Delta_{R} L_{R}+\lambda_{L} S \operatorname{Tr}\left[\Delta_{L} \delta_{L}\right]+\lambda_{R} S \operatorname{Tr}\left[\Delta_{R} \delta_{R}\right] \\
& +\lambda_{3} S \operatorname{Tr}\left[\tau_{2} \Phi_{1}^{T} \tau_{2} \Phi_{2}\right]+\lambda_{4} S \operatorname{Tr}\left[\tau_{2} \Phi_{1}^{T} \tau_{2} \Phi_{1}\right] \\
& +\lambda_{5} S \operatorname{Tr}\left[\tau_{2} \Phi_{2}^{T} \tau_{2} \Phi_{2}\right]+\lambda_{S} S^{3}+\xi_{F} S
\end{aligned}
$$

where the Yukawa couplings $\mathbf{Y}_{Q, L}$ and $\mathbf{h}_{L L, R R}$ are $3 \times 3$ matrices in the flavor space, and the $\lambda$ and $\xi_{F}$ parameters are associated with the various Higgs(ino) self-interactions. Moreover, in our notation, $\tau_{2}$ denotes the second Pauli matrix and we omit all indices for clarity. We derive from the form of the superpotential the corresponding soft terms, 
to which one should add scalar and gaugino mass terms. Further details of the model can be found in Refs. [18,33].

The superpotential possesses a $U(3, \mathbb{C})$ symmetry, whose spontaneous breaking leads to the appearance of several Goldstone bosons, one of them being the $S U(2)_{R}$ doubly charged Higgs state [14]. This doubly charged state remains massless even after the addition of soft SUSY breaking terms, and $D$-terms shifts its mass so that it is nearly always tachyonic. Loop corrections however restores a positive squared mass $[14,16]$. As the bulk of the mass is loop-induced, the $S U(2)_{R}$ doubly charged Higgs boson lies always in the lightest part of the particle spectrum, in contrast with any other of the numerous Higgs states of the model. Searches for the $S U(2)_{R}$ doubly charged Higgs boson are therefore promising in either discovering LRSUSY-related new physics, or excluding a large part of the parameter space.

The LRSUSY spectrum is largely determined by the scale of the left-right symmetry breaking. While current experimental limits imply that this scale has to lie in the multi-TeV range, it must at the same time satisfy an upper limit, so that $\left\langle\Delta_{R}^{0}\right\rangle \sim v_{R} \in[10,15] \mathrm{TeV}$ when all other parameters are held fixed [16]. Larger $v_{R}$ values would indeed destabilize the charge-conserving vacuum configuration, as the scalar potential terms

$$
\begin{aligned}
& \mid \xi_{F}+\lambda_{L} \operatorname{Tr}\left[\Delta_{L} \delta_{L}\right]+\lambda_{R} \operatorname{Tr}\left[\Delta_{R} \delta_{R}\right]+\lambda_{3} \operatorname{Tr}\left[\tau_{2} \Phi_{1}^{T} \tau_{2} \Phi_{2}\right] \\
& \quad+\lambda_{4} \operatorname{Tr}\left[\tau_{2} \Phi_{1}^{T} \tau_{2} \Phi_{1}\right]+\lambda_{5} \operatorname{Tr}\left[\tau_{2} \Phi_{2}^{T} \tau_{2} \Phi_{2}\right]+\left.3 \lambda_{S} S^{2}\right|^{2}
\end{aligned}
$$

would lift the energy of the corresponding ground state. However, if $v_{R}$ and $\langle S\rangle=v_{S} / \sqrt{2}$ are of the same order of magnitude and $\lambda_{R}$ and $\lambda_{S}$ are of opposite signs, this contribution can be kept small. In this small part of the parameter space, one may have values of $v_{R}$ clearly greater than $15 \mathrm{TeV}$, so that one gets a decoupling limit in which many particles become heavy due to the large $v_{R}$ and $v_{S}$ VEVs, unless one invokes unforeseen fine-tuning effects. In this limit, the $S U(2)_{R}$ gauge sector is easily beyond the reach of the LHC, so that the light part of the entire LRSUSY spectrum may only include an $S U(2)_{R}$ doubly charged Higgs state, as its mass is loop-suppressed relatively to the scale of left-right symmetry breaking, in addition to the LSP, the dark matter candidate.

In the following, we assume that the LSP belongs to the bidoublet Higgsino state, so that extra neutralinos and charginos are expected to be not too heavy as well. In this setup, a relic density in agreement with the observations can be achieved if the Higgsino spectrum lies below $1 \mathrm{TeV}$. The only HL-LHC handle on the model is then comprised of a signature made of a multileptonic system and missing energy that emerges from resonant Higgsino production (via the $S U(2)_{R}$ gauge sector) [19]. Other cosmologically favored options could feature the lightest right-handed sneutrino as a dark matter candidate. The spectrum does not significantly differ here from the Higgsino dark matter case, as to explain the nonobservation of any signal in the direct detection experiments and to avoid dark matter over-abundance, one needs to rely on the existence of co-annihilation channels [19]. This leads to a spectrum including a rather light sneutrino in addition to a set of light Higgsino states. If the $W_{R}$ gauge boson is too heavy to be produced at the LHC, the sneutrino signal will emerge either through multileptonic Higgsino cascade decays, as in the former Higgsino dark matter case, or with a different kinematic topology making it hard to access via traditional searches [34]. If the $W_{R}$ boson lies instead within the reach of the HL-LHC, we should expect multileptonic signals to originate from its decays into sleptons [18].

The light doubly charged Higgs state could then be the best probe of the model, as suggested by recent studies on the sensitivity of future colliders in the framework of Type-II seesaw and left-right models [35-39]. However, in LRSUSY and in contrast with Type-II seesaw scenarios, the couplings of the Higgs triplet are not determined by the neutrino masses and mixings, as the neutrino mass generation mechanism is comprised of a combination of Type-I and Type-II seesaws. In this case, right-handed neutrinos indeed get their mass through their interactions with the $\Delta_{R}$ weak triplet, which then opens neutrino mass generation through the usual Type-I seesaw.

The light neutrino mass matrix is approximately given by

$$
\left(m_{\nu}\right)_{i l}=\frac{v_{L}^{2}}{2 v_{R}}\left(\mathbf{Y}_{L}^{(2)}\right)_{i j}\left(\mathbf{h}_{R R}^{-1}\right)_{j k}\left(\mathbf{Y}_{L}^{(2)}\right)_{k l}^{\dagger}
$$

where $v_{R}\left(v_{u}\right)$ stands for the $S U(2)_{R}$ triplet (bidoublet) vacuum expectation value. Even if we choose $\mathbf{h}_{R R}$ freely, there are still enough freedom in the $\mathbf{Y}_{L}^{(2)}$ matrix so that it is possible to reproduce the observed neutrino masses and mixings.

This means that the $S U(2)_{R}$ doubly charged Higgs boson could dominantly decay into a pair of same-sign tau leptons, taming the sensitivity of the usually considered same-sign electron or muon channels. In addition, as the doubly charged Higgs state belongs to an $S U(2)_{R}$ triplet and not an $S U(2)_{L}$ triplet as in the type-II case, the production mechanisms are related to different gauge interactions. Moreover, the much heavier $S U(2)_{R}$ singly charged Higgs bosons tame the potential relevance of associated production down, again in contrast with what typically occurs for $S U(2)_{L}$ triplets.

In the rest of this work, we focus on such LRSUSY scenarios in which the Yukawa couplings responsible for the right-handed neutrino masses obey the same generational hierarchy as for the other SM fermions, i.e., the coupling to the third generation is the largest. The $S U(2)_{R}$ doubly charged Higgs boson then features a main decay mode into tau leptons, and it could be light without violating any LHC constraint. Moreover, the lightest superpartners are the neutral and charged bidoublet Higgsinos, 
the lightest one being neutral and a viable dark matter candidate. Such an LRSUSY configuration would be experimentally the most challenging to observe, and therefore deserves the present dedicated study.

\section{COLLIDER PHENOMENOLOGY}

\section{A. Generalities}

As detailed in the previous section, the class of LRSUSY scenarios that we consider focuses on setups in which the $S U(2)_{R}$ doubly charged Higgs boson $\left(H_{1}^{ \pm \pm}\right)$is relatively light $[18,19]$. In the minimal version of the model, its mass cannot be much larger than $1 \mathrm{TeV}[16,40]$. The $H_{1}^{ \pm \pm}$boson can thus in principle be reachable at the $14 \mathrm{TeV}$ LHC, even without the need of a very high luminosity.

The derivation of the current limits on the $S U(2)_{R}$ doubly charged Higgs bosons requires some careful interpretation within the LRSUSY context. The ATLAS collaboration has searched for doubly charged scalars that decay to electrons or muons [41] after being produced through the Drell-Yan mechanism (that is the dominant production mode in LRSUSY models). The limits read

$$
m_{H_{1}^{ \pm \pm}} \gtrsim 650 \mathrm{GeV}
$$

the exact bounds depending on the precise values of the $H_{1}^{ \pm \pm}$ branching ratios in electrons and muons. Branching ratios as low as $\operatorname{BR}\left(H_{1}^{ \pm \pm} \rightarrow \ell^{ \pm} \ell^{ \pm}\right)=4 \%$ (for $\ell=e$ or $\mu$ ) are required to avoid most bounds, so that $m_{H_{1}^{ \pm \pm}} \gtrsim 350 \mathrm{GeV}$.

The limits consequently become much weaker as soon as the $H_{1}^{ \pm \pm}$state dominantly decays into a pair of tau leptons. The corresponding search has been performed by the CMS collaboration [42], both for a setup in which the doubly charged Higgs boson is pair produced and when it is produced in association with a singly charged Higgs boson. In LRSUSY models, singly charged Higgs bosons are typically a lot heavier than the doubly charged ones, so that they can be ignored from the discussion. In the case of a branching ratio $\operatorname{BR}\left(H_{1}^{ \pm \pm} \rightarrow \tau^{ \pm} \tau^{ \pm}\right)=100 \%$, the results lead to $m_{H_{1}^{ \pm \pm}} \geq 396 \mathrm{GeV}$ for $S U(2)_{L}$ doubly charged Higgs bosons and slightly weaker bounds for $S U(2)_{R}$ ones.

The most difficult combination would hence result from a scenario in which $\operatorname{BR}\left(H_{R}^{ \pm \pm} \rightarrow \tau^{ \pm} \tau^{ \pm}\right)=92 \%$ and $\mathrm{BR}\left(H_{R}^{ \pm \pm} \rightarrow \mu^{ \pm} \mu^{ \pm}\right)=\mathrm{BR}\left(H_{R}^{ \pm \pm} \rightarrow e^{ \pm} e^{ \pm}\right)=4 \%$. In this case, we estimate that

$$
m_{H_{1}^{ \pm \pm}} \gtrsim 350 \mathrm{GeV}
$$

Under these circumstances, even HL-LHC operations are unlikely to be effective in probing heavier $H_{1}^{ \pm \pm}$ bosons, especially if their mass gets close to $1 \mathrm{TeV}$. This is the type of complicated scenarios that we are interested in in this work. We hence aim at estimating the prospects of a potential high-energy upgrade of the LHC at a center-of-mass energy of $27 \mathrm{TeV}$ to probe scenarios in which $H_{1}^{ \pm \pm} \rightarrow \tau^{ \pm} \tau^{ \pm}$is the dominant decay mode.

We consider the all-hadronic channel for which no dedicated study exists. In our analysis, we first design a signal region SR1 targeting a signature arising from the production of a pair of $H_{1}^{ \pm \pm}$bosons decaying each into a ditau system. In other words, we focus on a final state featuring four hadronic tau leptons $\tau_{h}$. We however only select events in which three hadronic taus have been reconstructed, which guarantees both sufficient signal rates after accounting for imperfect tau reconstruction, and a not too overwhelming SM background. Moreover, we additionally require some missing transverse energy as it would stem from the tau decays.

Furthermore, we also explore how the subleading $H_{1}^{ \pm \pm}$ branching ratio into same-sign electron or muon pairs could be used to get an extra handle on the signal, as the corresponding signature is cleaner to reconstruct. We define a second signal region SR2 in which one selects events featuring a same-sign lepton (i.e., electron or muon) pair and a di-tau system.

We therefore consider the following two signal regions,

$$
\begin{aligned}
& \text { SR1: At least } 3 \tau_{h} \text {, some } \mathscr{E}_{T}^{\dagger} \text {; } \\
& \text { SR2: At least } 2 \tau_{h}, 1 \text { same-sign lepton pair, some } \mathscr{E}_{T}^{\dagger} \text {. }
\end{aligned}
$$

In order to generate signal events at the HE-LHC for given benchmark scenarios, we make use of the LRSUSY model implementation in the SARAH package $[16,43]$. This allows for both the computation of the particle spectrum and branching ratios through SPHENO3 [44], and for the translation of the model into the UFO format [45] so that hard-scattering event generation could be achieved with MG5_AMC@NLO [46]. For both signal and background, we convolute leading-order matrix elements with the NNPDF 2.3 set of parton distribution functions [47] and match the resulting events with the parton showering and hadronization infrastructure of PYTHIA8 [48]. Subsequently, we implement the simulation of the detector response with DELPHES3 [49], that relies on the anti- $k_{T}$ algorithm [50] as implemented in the FASTJET package [51] for event reconstruction, and use the default ATLAS detector parametrization. For a better description of the background, we merge multipartonic matrix elements according to the MLM procedure [52], unless stated otherwise. Finally, in order to obtain cosmologically favored benchmark scenarios (see Sec. III B), we estimate the dark matter properties of our scenarios with the MADDM package [53].

\section{B. Benchmark scenarios}

In order to assess the sensitivity of the HE-LHC to the considered class of LRSUSY scenarios, we select three representative benchmark configurations BP1, BP2, and BP3 featuring a different $S U(2)_{R}$ doubly charged Higgs 
TABLE I. Relevant masses defining our three benchmark scenarios, given in $\mathrm{GeV}$, and $H_{1}^{ \pm \pm}$branching ratios. The table includes the masses of the $W_{R}$ and $Z^{\prime}$ extra gauge bosons, the one of the $H_{1}^{ \pm \pm}$state, those of the lightest neutralino $\tilde{\chi}_{i}^{0}$ (with $i=1,2$, 3,4 ), singly charged charginos $\tilde{\chi}_{i}^{ \pm}$(with $i=1,2$ ) and doubly charged chargino $\tilde{\chi}_{1}^{ \pm \pm}$.

\begin{tabular}{lrrr}
\hline \hline & \multicolumn{1}{c}{$\mathrm{BP} 1$} & \multicolumn{1}{c}{$\mathrm{BP} 2$} & \multicolumn{1}{c}{$\mathrm{BP3}$} \\
\hline$m_{W_{R}}$ & 6550.5 & 7486.2 & 7486.2 \\
$m_{Z^{\prime}}$ & 10993.2 & 12563.6 & 12563.6 \\
$m_{H_{1}^{ \pm \pm}}$ & 875.0 & 1016.4 & 780.9 \\
$m_{\tilde{\chi}_{1}^{0}}$ & 878.4 & 803.7 & 770.1 \\
$m_{\tilde{\chi}_{2}^{0}}$ & 889.7 & 812.1 & 777.7 \\
$m_{\tilde{\chi}_{3}^{0}}$ & 893.0 & 815.3 & 780.7 \\
$m_{\tilde{\chi}_{4}^{0}}$ & 895.6 & 817.6 & 782.9 \\
$m_{\tilde{\chi}_{5}^{0}}$ & 1032.2 & 1043.5 & 1048.0 \\
$m_{\tilde{\chi}_{1}^{ \pm}}$ & 886.4 & 809.3 & 775.1 \\
$m_{\tilde{\chi}_{2}^{ \pm}}$ & 893.5 & 815.7 & 781.2 \\
$m_{\tilde{\chi}_{1}^{ \pm \pm}}$ & 7413.2 & 8412.9 & 5619.2 \\
$\mathrm{BR}\left(H_{1}^{ \pm \pm} \rightarrow \tau^{ \pm} \tau^{ \pm}\right)$ & 0.92 & 0.92 & 0.92 \\
$\mathrm{BR}\left(H_{1}^{ \pm \pm} \rightarrow \ell^{ \pm} \ell^{ \pm}\right)$ & 0.08 & 0.08 & 0.08 \\
\hline \hline
\end{tabular}

boson mass. The LSP is enforced to be part of the Higgsino bidoublets and its mass and properties are constrained to lead to a cosmologically viable dark matter candidate. Its relic density is required to agree with latest Planck data [54], which can be achieved thanks to multiple co-annihilation processes among the six nearly mass-degenerate Higgsino-like neutralino and chargino states [19]. The light part of the benchmark scenario spectra is presented in Table I, together with the relevant branching ratios of the $H_{1}^{ \pm \pm}$state.

We choose the Yukawa coupling matrix $h_{R R}$ to be diagonal and yield branching ratios $\operatorname{BR}\left(H_{R}^{ \pm \pm} \rightarrow \tau^{ \pm} \tau^{ \pm}\right)=$ $92 \%$ and $\operatorname{BR}\left(H_{R}^{ \pm \pm} \rightarrow \mu^{ \pm} \mu^{ \pm}\right)=\mathrm{BR}\left(H_{R}^{ \pm \pm} \rightarrow e^{ \pm} e^{ \pm}\right)=4 \%$. As said above, this combination is the most challenging one with respect to the searches. For instance, when the decay rate to one of the lighter leptons is larger, stronger bounds immediately arise from the light lepton channels.

\section{SR1: Investigating the triple-tau signature}

Our SR1 signal region focuses on events featuring at least three reconstructed hadronic tau leptons and missing energy. SM backgrounds can arise from QCD multijet processes and single boson production in association with jets $\left(V+\right.$ jets, with $\left.V \equiv W^{ \pm}, Z\right)$ when light jets are mistagged as hadronic taus. We study, in our analysis, the dependence of the results on the tau mistagging rate, that is allowed to vary between $1 \%$ and $2 \%$. This choice stems from the absence of any realistic mistagging rate expectation for a potential future proton-proton collider at a center-of-mass energy of $27 \mathrm{TeV}$ [20], and has been inspired by LHC capabilities for a tau-tagging efficiency of 70\% [55,56]. Moreover, one expects subleading background contributions originating from $Z h, h h, V V$ and $V V V$ production in association with jets. For each component of the background and all signal samples, the tautagging performances are included in the evaluation of the rates presented in the following.

In order to avoid excessive multi-jet and $V+$ jets background event generation (to get numerical Monte Carlo uncertainties under control after accounting for the small mistagging rates), we make use of the properties of the signal in which quite hard tau jets with a large transverse momentum $p_{T}$ should originate from the $H_{1}^{ \pm \pm}$decays. We implement a set of generator-level cuts (consistent with the subsequent analysis) while simulating those background components, and hence require the transverse momentum of all (parton-level) jets to satisfy $p_{T}>40 \mathrm{GeV}$.

In the multijet case, we increase this selection criterion to $p_{T}>150 \mathrm{GeV}$ for the three hardest jets and impose that the hadronic activity defined as the sum of the transverse momenta of all reconstructed jets is greater than $1200 \mathrm{GeV}$. In practice, we separately generate hard-scattering events for the $p p \rightarrow j j j$ and $p p \rightarrow j j j j$ subprocesses and match them with parton showers, the dijet case being ignored given the need for the events selected in our analysis to feature at least three hard jets faking tau leptons. Whilst in principle the overlap between the trijet and tetrajet samples should be removed by an appropriate merging procedure, we instead directly combine those two samples for simplicity. This is expected to yield a (conservative) overestimation of the multijet background.

For $V+$ jets event generation, we similarly separately consider the $V+j j$ and $V+j j j$ subprocesses and conservatively directly combine them. The overlap between the two samples is however expected to be small, as any extra radiation originating from a $V+j j$ final state is generally soft. All other background components are treated as described in Sec. III A.

In the considered SR1 signal region, one preselects events by requiring that they feature at least three hadronic taus $\tau_{i}$ (or jets faking taus) with a transverse momentum

$$
p_{T}\left(\tau_{i}\right)>150 \mathrm{GeV}
$$

We moreover veto the presence of any charged lepton with a $p_{T}$ greater than $20 \mathrm{GeV}$ and of any $b$-tagged jet with a $p_{T}$ greater than $25 \mathrm{GeV}$. Moreover, any system comprised of any two hadronic tau $\left(\tau_{i}, \tau_{j}\right)$ must have an invariant mass satisfying

$$
m_{\tau_{i} \tau_{j}}>200 \mathrm{GeV} \text { for } i, j=1,2,3 \text {. }
$$

After this preselection, we impose that the scalar sum of the transverse momentum of the three leading taus (including the potential jets faking taus) satisfies 
TABLE II. Cross sections, in fb, for the three benchmark signals and the different components of the SM background at various stages of the SR1 analysis. We present generator-level total rates (second column), as well as the reminding cross sections after the preselection (third column) and the various analysis cuts of Eq. (10), Eq. (11) and Eq. (12) (last three columns). We consider a 70\% tau-tagging efficiency for a mistagging rate of $1 \%$. Results for a mis-tagging rate of $2 \%$ are given between parentheses (where relevant).

\begin{tabular}{lccccc}
\hline \hline Process & Generator & Preselection & $H_{T}>1200 \mathrm{GeV}$ & $E_{T}>150 \mathrm{GeV}$ & $\mathcal{S}>0.3$ \\
\hline BP1 & 0.251 & 0.020 & 0.013 & 0.010 & 0.006 \\
BP2 & 0.125 & 0.011 & 0.008 & 0.007 & 0.004 \\
BP3 & 0.430 & 0.031 & 0.017 & 0.014 & 0.008 \\
$p p \rightarrow V V+$ jets & $3.08 \times 10^{5}$ & $7.0 \times 10^{-4}(0.0056)$ & $2.5 \times 10^{-4}(0.002)$ & $\sim 10^{-5}$ & $\sim 10^{-5}$ \\
$p p \rightarrow W^{ \pm}+$jets & $1.31 \times 10^{7}$ & $0.065(0.520)$ & $0.014(0.112)$ & $0.003(0.024)$ & $14.7 \times 10^{-4}(0.011)$ \\
$p p \rightarrow Z+$ jets & $4.36 \times 10^{6}$ & $0.0206(0.165)$ & $0.004(0.032)$ & $14.2 \times 10^{-4}(0.011)$ & $5.0 \times 10^{-4}(0.004)$ \\
$p p \rightarrow$ jets & $6.42 \times 10^{6}$ & $1.313(10.504)$ & $0.913(7.304)$ & $0.054(0.432)$ & $0.016(0.128)$ \\
\hline \hline
\end{tabular}

$$
H_{T}=\sum_{i=1}^{3} p_{T}\left(\tau_{i}\right)>1200 \mathrm{GeV}
$$

and require that the missing transverse energy fulfills

$$
E_{T}>150 \mathrm{GeV}
$$

Finally, in order to ensure a better rejection of the multijet background, we require the event sphericity $\mathcal{S}$ [57], computed from the three selected taus, to be larger than 0.3 ,

$$
\mathcal{S}>0.3
$$

The corresponding cutflow is provided, for the three benchmark points and all the components of the background, in Table II. As the tau-jets arising from the on-shell decays of weak gauge and Higgs bosons are typically softer than in the case of our signal, the corresponding multiboson backgrounds are drastically rejected already by our preselection cuts. The dominant background components are therefore driven by jets faking hadronic tau leptons. These can however be significantly reduced by the three extra cuts of Eq. (10), Eq. (11) and Eq. (12), for a moderate signal efficiency of about 50\%. As shown in Table III, about $6 \mathrm{ab}^{-1}, 12.4 \mathrm{ab}^{-1}$ and $3.7 \mathrm{ab}^{-1}$ of data would be needed for a $3 \sigma$ statistical significance in the BP1, BP2 and BP 3 cases, respectively, three luminosities that are well within the reach of the HE-LHC, which is indeed expected to collect a luminosity as high as $15 \mathrm{ab}^{-1}$.

TABLE III. Required luminosities, in $\mathrm{ab}^{-1}$, to obtain a $3 \sigma$ statistical significance with our cut-and-count (second column) and multi-variate (third column) analysis, for the three considered benchmark scenarios.

\begin{tabular}{lcc}
\hline \hline Scenario & Cut-and-count & Multivariate \\
\hline BP1 & 6.0 & 1.95 \\
BP2 & 12.38 & 3.91 \\
BP3 & 3.66 & 1.14 \\
\hline \hline
\end{tabular}

Given the low sensitivity of the previously described cutbased analysis, we implement a complementarily multivariate analysis based on a boosted decision tree (BDT). We first preselect events as described in the cut-and-count analysis and impose the $H_{T}$ selection of Eq. (10). We then rely on seven variables as inputs for our BDT classifier, namely the invariant mass of any system made of any pair of two of the three leading taus $m_{\tau_{i} \tau_{j}}$ (with $i, j=1,2,3$ ), the missing transverse energy $\mathscr{E}_{T}$, the effective mass $M_{\text {eff }}$ defined as

$$
M_{\mathrm{eff}}=\sum_{i=1,3} p_{T}\left(\tau_{i}\right)+\not \mathscr{E}_{T}
$$

the angular separation in azimuth between the missing transverse momentum $\not \not_{T}$ and the leading tau $\Delta \phi\left(\not \not_{T}, \mathbf{p}_{T}\left(\tau_{1}\right)\right)$ and the sphericity $\mathcal{S}$. We extract the HE-LHC sensitivity by using the XGBOOST toolkit [58], employing the gradient boosting method with a number of 1000 trees, a maximum depth of 4 and a learning rate of 0.01 . Our training set includes $80 \%$ of the generated events, the remaining events being then used for testing purposes. Moreover, we have verified that our results were not affected by the addition of an extra variable to the list of BDT inputs, both in the context of basic quantities like the transverse momentum of any of the three leading taus or their azimuthal separation, and in the context of more complex observables like the aplanarity $A$ [57], the missing energy significance $E_{T} / \sqrt{H_{T}}$, the $E_{T}^{\prime} / M_{\text {eff }}$ ratio, or the relative $p_{T}$ of the third tau with respect to the two leading ones $y_{23}$,

$$
y_{23}=\frac{p_{T}^{2}\left(\tau_{3}\right)}{\left[p_{T}\left(\tau_{1}\right)+p_{T}\left(\tau_{2}\right)\right]^{2}} .
$$

Including any extra variable on top of the seven abovementioned ones has indeed only been found to increase the correlations. While the relative relevance of the variables varies from benchmark to benchmark, the level 


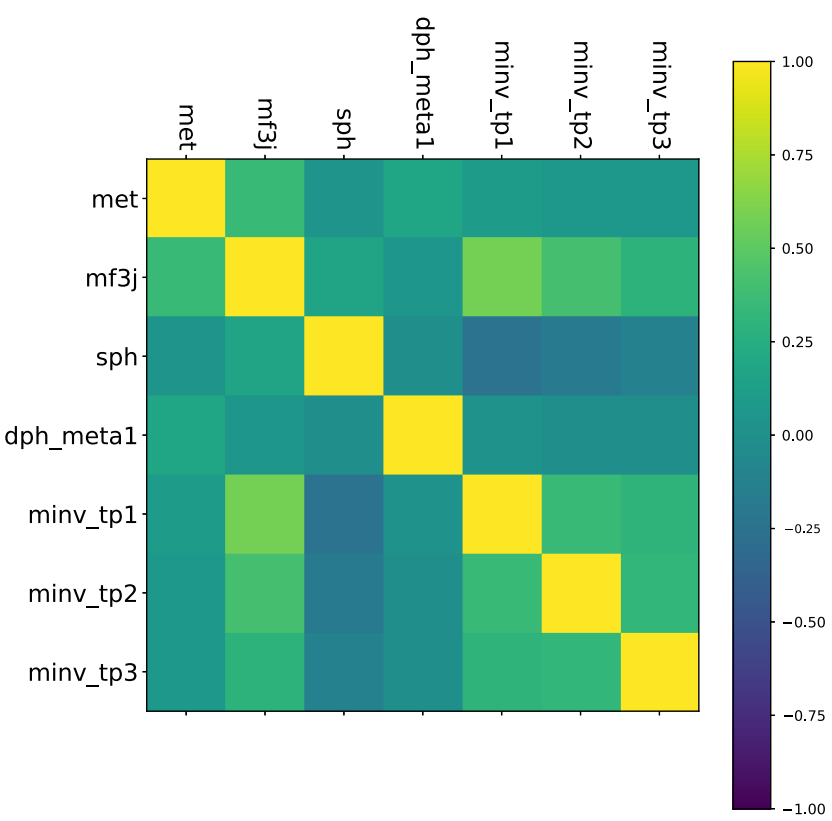

FIG. 1. Correlations among the seven essential kinematic variables used in our multi-variate analysis of the BP1 doubly charged Higgs boson signal. The labels of the $x$-axis and $y$-axis refer, from the first to the last variable, to $E_{T}, M_{\text {eff }}, \mathcal{S}$, $\Delta \phi\left(\not p_{T}, \mathbf{p}_{T}\left(\tau_{1}\right)\right), m_{\tau_{1} \tau_{2}}, m_{\tau_{1} \tau_{3}}$ and $m_{\tau_{2} \tau_{3}}$, respectively.

of correlations is maintained in each case to a low level, as illustrated in Fig. 1 for the BP1 scenario.

As evident from Fig. 2 for the BP1 scenario, our BDT classifier is quite efficient in identifying signal events whilst rejecting background events, as signal efficiencies larger than $80 \%$ can be obtained together with high background rejection rates. In the upper panel of the figure, we present the receiver operating characteristic (ROC) curve of the algorithm. The area under the curve (AUC) is a good indicator of the algorithm effectiveness as it should approach 1 for wellperforming methods. It is found to be of about 0.92 for the BP1 scenario, whilst similar results are obtained for the BP2 and $\mathrm{BP} 3$ scenarios, with AUC of 0.88 and 0.82 respectively. In the lower panel of Fig. 2, we present the signal and background distributions (in the case of the BP1 scenario) according to the BDT classifier, which reinforces the illustration of its good discriminating power. This largely impacts the sensitivity of the HE-LHC to the model, the resulting significance factors being importantly improved relatively to the cut-based analysis. This is complementarily demonstrated in Table III in which the HE-LHC luminosity needed to reach a $3 \sigma$ statistical significance is presented for each of our three representative scenarios. In all three cases, this luminosity is found three times smaller than for the cutbased analysis.

\section{SR2: Investigating the ditau plus dilepton signature}

In our three benchmark scenarios, the $H_{1}^{ \pm \pm}$branching ratio into a pair of same-sign electrons or muons has been
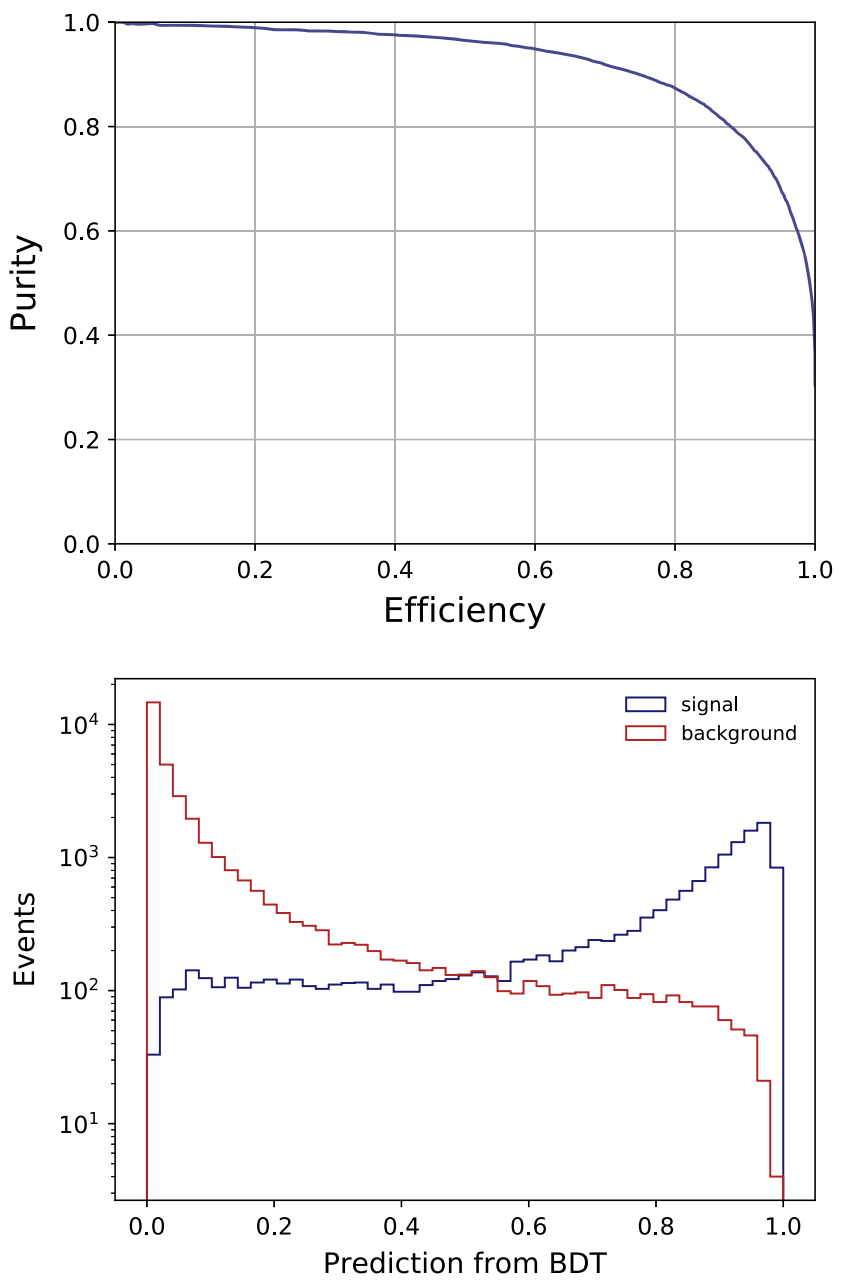

FIG. 2. Properties of our BDT classifier in the context of the BP1 scenario. We present the obtained receiver operating characteristic (ROC) curve (upper panel), in which the efficiency measures the fraction of identified signal events that would pass a selection on the classifier and the purity denotes the ratio of identified signal events passing this selection to the total number of identified signal and background events. In the lower panel, we show the normalized distribution in the classifier for the signal (blue) and the background (red).

fixed to $8 \%$ (see Table I), which is small enough to evade the current LHC constraints originating from doubly charged Higgs boson searches [18]. As a result, any handle on the model relying on the doubly charged Higgs boson decay into a same-sign-same-flavor lepton pair is associated with a quite small signal cross section. However, the requirement for the presence of these two leptons along two hard hadronic taus ensures that there is not much background surviving the selection.

In a first $\mathrm{C} 1$ preselection, we impose that events contain at least two hadronic taus with a transverse momentum $p_{T}>150 \mathrm{GeV}$ and we additionally constrain the invariant mass of the system made of the two hardest taus to be larger than $200 \mathrm{GeV}$. We moreover veto the presence of $b$-jets. 
TABLE IV. Cross sections in fb, for the signal as well as the most dominant background process after imposing the selection criteria for the SR2 signal region. We follow the same convention as in Table II.

\begin{tabular}{lcc}
\hline \hline Process & $\mathrm{C} 1$ & $\mathrm{C} 2$ \\
\hline BP1 & 0.094 & 0.003 \\
BP2 & 0.050 & 0.002 \\
BP3 & 0.150 & 0.005 \\
$p p \rightarrow V V+$ jets & $0.238(0.952)$ & $10^{-4}\left(4 \times 10^{-4}\right)$ \\
\hline \hline
\end{tabular}

We then add a $\mathrm{C} 2$ selection in which we focus on events featuring a pair of same-sign electrons or muons with $p_{T}>50 \mathrm{GeV}$. With these criteria, the SM background contribution is rendered negligible, with a cross section of about $10^{-4} \mathrm{fb}$ after including the $Z h, V V+$ jets, $V V V$ and $V+$ jets components of the background. After the lepton requirements $\mathrm{C} 2$, only $V V+$ jets events in fact contribute to the background. The signal rates in the context of the three considered benchmark scenarios and the most dominant background channel are presented in Table IV. We equivalently obtain an almost backgroundfree environment for a handful of signal events for the expected $15 \mathrm{ab}^{-1}$ luminosity of the HE-LHC.

As a consequence, the SR2 analysis may provide a complementary handle on the signal, relatively to the SR1 one, with an enormous advantage in the fact that the doubly charged Higgs-boson mass can be reconstructed from the analysis of the properties of the pair of same-sign leptons (that moreover consists in a smoking gun signal for a doubly charged Higgs boson). This mass reconstruction can be quite precise despite the detector

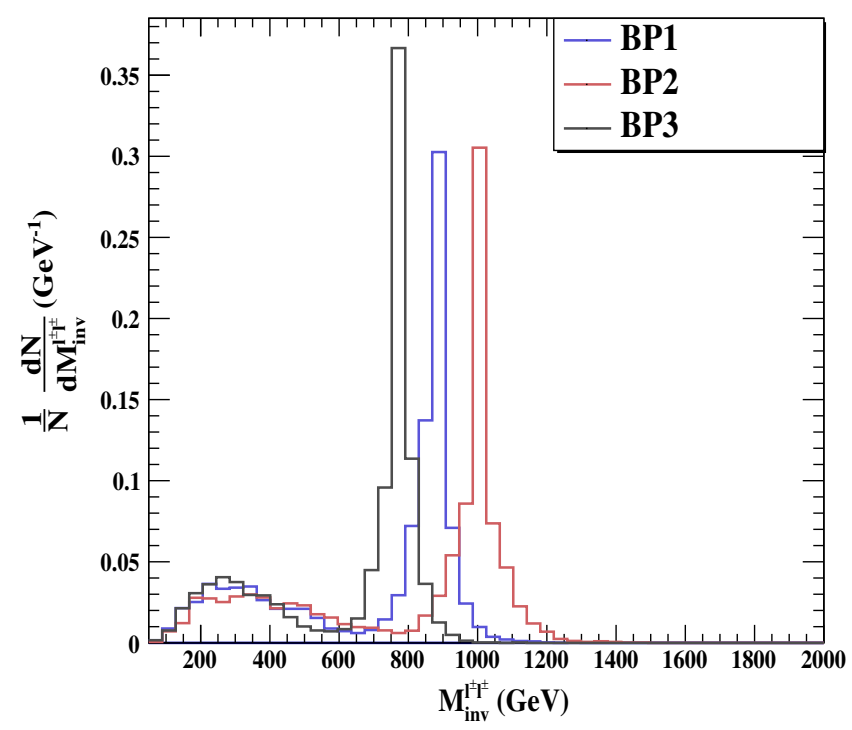

FIG. 3. Distribution in the invariant mass of the same-signsame-flavor lepton pair originating from the $H_{1}^{ \pm \pm}$decay for the three chosen benchmark points, after imposing the SR2 selection. effects, as illustrated in Fig. 3. In this figure, we present the invariant-mass spectrum of the dilepton-pair for all three benchmark scenarios. In each case, the distribution exhibits a clear peak located right at the doubly charged Higgsboson mass.

Apart from the two signal regions defined in this work, one can also build an analysis targeting a signature stemming from the production of multiple Higgsino states. Altogether, the considered class of scenarios features spectra in which four neutralino and two chargino states are nearly mass degenerate and sitting at the lighter part of the LRSUSY model particle spectrum. However, as a consequence, any SM jets and/or leptons that may arise from Higgsino production and decay is expected to be too soft to be detected. The standard probe to such scenarios consists thus of the monojet channel, that is at least promising in MSSM-like scenarios. In this last case, Higgsino masses of $500 \mathrm{GeV}$ can be reached at the HELHC [59]. Owing to a richer Higgsino sector in the LRSUSY framework, one can expect a larger signal cross section for a fixed mass, so that heavier Higgsino states could conversely be probed. However, the lower limit on the Higgsino mass, so that we could obtain a viable dark matter candidate is of about $700 \mathrm{GeV}$ [19]. The corresponding monojet rates have been found to be subsequently too low to lead to any observable signal with $15 \mathrm{ab}^{-1}$ of HE-LHC luminosity.

\section{SUMMARY AND CONCLUSION}

We have analyzed the sensitivity of a high-energy upgrade of the LHC expected to operate at a center-ofmass energy of $27 \mathrm{TeV}$ (i.e., the HE-LHC) to a class of leftright supersymmetric scenarios favored by dark matter, with a relic density as measured by the Planck collaboration originating from the coannihilations of multiple Higgsino states of about $700 \mathrm{GeV}$. In a minimal LRSUSY setup where the stabilization of the vacuum occurs through radiative corrections, the doubly charged Higgs boson mass is loop-suppressed relatively to the rest of the $S U(2)_{R}$ sector, so that we expect it to be the first manifestation of the model at colliders. As previous experimental limits on this state are obtained by assuming a pair-production mode followed by a decay into a same-sign pair of electrons or muons, we focus on the still phenomenologically viable option where the doubly charged Higgs boson decays almost exclusively into tau leptons. We explore this possibility and estimate the chances to observe such an LRSUSY scenario at the HE-LHC.

We consider in particular two signatures, namely a first one where the pair-produced doubly charged Higgs states decay into tau leptons, and a second one in which one of them is assumed to decay into an electron or a muon pair. For the former case, we focus on the production of at least three hard hadronic tau leptons. The SM background is mostly comprised of multijet and vector-boson-plus-jets 
events in which QCD jets are faking tau leptons. We have implemented a series of cuts which not only lead to a good significance at high luminosities, but that can also serve as a basis for a multivariate analysis relying on boosted decision trees. In this case, three times less luminosity could be required to observe a $3 \sigma$ signal (which would occur thus at an early HE-LHC stage). For our latter analysis, we position ourselves in an almost backgroundfree environment by investigating a ditau plus same-sign dilepton signature. Whereas signal cross sections are expected to be small, the large HE-LHC luminosity makes this analysis a nice complementary handle on the previously considered LRSUSY signal. Moreover, the presence of the two first or second generation leptons guarantees the reconstruction of the doubly charged Higgs boson mass. In contrast, any signal that could arise from the large number of light Higgsino states has been found not to give any hope for a discovery, as the corresponding monojet cross sections are way too small.

In summary, in the minimal left-right supersymmetry setup in which one relies on radiative corrections to stabilize the vacuum configuration, we expect the first signal of new physics to arise from the doubly charged Higgs boson. However, it may hidden as decaying mainly into a pair of same-sign tau leptons. We have shown that at the HE-LHC, we may see a signal for doubly charged Higgs boson masses ranging up to around $1 \mathrm{TeV}$, this upper limit being theoretically motivated as it requires to push the left-right symmetry breaking scale in a way that is only possible in a very small part of the parameter space. In addition to the doubly charged Higgs boson, the model includes a dark matter candidate that cannot be too heavy. The latter will however hardly provide any clear signal without the help of the $S U(2)_{R}$ gauge sector, as shown in previous work.

If the doubly charged Higgs boson fails to materialize at the HE-LHC, we may conclude that the LRSUSY vacuum has likely to be stabilized by some other mechanism than by loop corrections. Models employing an even larger Higgs sector or those breaking the $R$-parity spontaneously may have a tree-level contribution to the doubly charged Higgs boson mass, which could thus be larger.

\section{ACKNOWLEDGMENTS}

M.F. thanks NSERC for support through Grant No. SAP105354. S. K. R. acknowledges financial support from the Department of Atomic Energy, Government of India, for the Regional Centre for Accelerator-based Particle Physics (RECAPP), Harish-Chandra Research Institute. H. W. acknowledges the support from Magnus Ehrnrooth Foundation and STFC Rutherford International Fellowship (funded through MSCA-COFUND-FP, Grant No. 665593).
[1] H. P. Nilles, Phys. Rep. 110, 1 (1984).

[2] H. E. Haber and G. L. Kane, Phys. Rep. 117, 75 (1985).

[3] J. C. Pati and A. Salam, Phys. Rev. D 10, 275 (1974); 11, 703(E) (1975).

[4] R. N. Mohapatra and J. C. Pati, Phys. Rev. D 11, 566 (1975).

[5] R. N. Mohapatra and J. C. Pati, Phys. Rev. D 11, 2558 (1975).

[6] R. N. Mohapatra and A. Rasin, Phys. Rev. Lett. 76, 3490 (1996).

[7] R. Kuchimanchi, Phys. Rev. Lett. 76, 3486 (1996).

[8] R. N. Mohapatra and A. Rasin, Phys. Rev. D 54, 5835 (1996).

[9] M. Cvetic and J. C. Pati, Phys. Lett. 135B, 57 (1984).

[10] A. M. Sirunyan et al. (CMS Collaboration), Phys. Lett. B 769, 520 (2017); 772, 882(E) (2017).

[11] M. Aaboud et al. (ATLAS Collaboration), Phys. Rev. D 96, 052004 (2017).

[12] M. Cvetic, Phys. Lett. 164B, 55 (1985).

[13] C. S. Aulakh, A. Melfo, and G. Senjanovic, Phys. Rev. D 57, 4174 (1998).

[14] K. S. Babu and R. N. Mohapatra, Phys. Lett. B 668, 404 (2008).

[15] M. Frank and B. Korutlu, Phys. Rev. D 83, 073007 (2011).
[16] L. Basso, B. Fuks, M. E. Krauss, and W. Porod, J. High Energy Phys. 07 (2015) 147.

[17] R. Kuchimanchi and R. N. Mohapatra, Phys. Rev. D 48, 4352 (1993).

[18] M. Frank, B. Fuks, K. Huitu, S. K. Rai, and H. Waltari, J. High Energy Phys. 05 (2017) 015.

[19] A. Chatterjee, M. Frank, B. Fuks, K. Huitu, S. Mondal, S. K. Rai, and H. Waltari, Phys. Rev. D 99, 035017 (2019).

[20] A. Abada et al. (FCC Collaboration), Eur. Phys. J. Special Topics 228, 1109 (2019).

[21] M. Magg and C. Wetterich, Phys. Lett. 94B, 61 (1980).

[22] T. P. Cheng and L.-F. Li, Phys. Rev. D 22, 2860 (1980).

[23] R. N. Mohapatra and G. Senjanovic, Phys. Rev. D 23, 165 (1981).

[24] G. Lazarides, Q. Shafi, and C. Wetterich, Nucl. Phys. B181, 287 (1981).

[25] J. Schechter and J. W. F. Valle, Phys. Rev. D 22, 2227 (1980).

[26] Y. Cai, T. Han, T. Li, and R. Ruiz, Front. Phys. 6, 40 (2018).

[27] P. Minkowski, Phys. Lett. 67B, 421 (1977).

[28] T. Yanagida, Conf. Proc. C 7902131, 95 (1979), https:// inspirehep.net/literature/143150.

[29] M. Gell-Mann, P. Ramond, and R. Slansky, Conf. Proc. C 790927, 315 (1979), https://inspirehep.net/literature/9686.

[30] S. L. Glashow, NATO Sci. Ser. B 61, 687 (1980). 
[31] R. N. Mohapatra and G. Senjanovic, Phys. Rev. Lett. 44, 912 (1980).

[32] R. E. Shrock, Phys. Rev. D 24, 1232 (1981).

[33] A. Alloul, M. Frank, B. Fuks, and M. Rausch de Traubenberg, J. High Energy Phys. 10 (2013) 033.

[34] R. Ruiz, Eur. Phys. J. C 77, 375 (2017).

[35] T. B. de Melo, F. S. Queiroz, and Y. Villamizar, Int. J. Mod. Phys. A 34, 1950157 (2019).

[36] R. Padhan, D. Das, M. Mitra, and A. Kumar Nayak, Phys. Rev. D 101, 075050 (2020).

[37] B. Fuks, M. Nemevšek, and R. Ruiz, Phys. Rev. D 101, 075022 (2020).

[38] P. S. Bhupal Dev, R. N. Mohapatra, and Y. Zhang, J. High Energy Phys. 05 (2016) 174.

[39] P. S. Bhupal Dev, R. N. Mohapatra, and Y. Zhang, Phys. Rev. D 98, 075028 (2018).

[40] K. S. Babu and A. Patra, Phys. Rev. D 93, 055030 (2016).

[41] M. Aaboud et al. (ATLAS Collaboration), Eur. Phys. J. C 78, 199 (2018).

[42] CMS Collaboration (2017), https://cds.cern.ch/record/ $2242956 ? \ln =\mathrm{en}$.

[43] F. Staub, Comput. Phys. Commun. 185, 1773 (2014).

[44] W. Porod and F. Staub, Comput. Phys. Commun. 183, 2458 (2012).

[45] C. Degrande, C. Duhr, B. Fuks, D. Grellscheid, O. Mattelaer, and T. Reiter, Comput. Phys. Commun. 183, 1201 (2012).

[46] J. Alwall, R. Frederix, S. Frixione, V. Hirschi, F. Maltoni, O. Mattelaer, H. S. Shao, T. Stelzer, P. Torrielli, and M. Zaro, J. High Energy Phys. 07 (2014) 079.
[47] R. D. Ball et al. (NNPDF Collaboration), J. High Energy Phys. 04 (2015) 040.

[48] T. Sjöstrand, S. Ask, J. R. Christiansen, R. Corke, N. Desai, P. Ilten, S. Mrenna, S. Prestel, C. O. Rasmussen, and P.Z. Skands, Comput. Phys. Commun. 191, 159 (2015).

[49] J. de Favereau, C. Delaere, P. Demin, A. Giammanco, V. Lemaitre, A. Mertens, and M. Selvaggi (DELPHES 3 Collaboration), J. High Energy Phys. 02 (2014) 057.

[50] M. Cacciari, G. P. Salam, and G. Soyez, J. High Energy Phys. 04 (2008) 063.

[51] M. Cacciari, G. P. Salam, and G. Soyez, Eur. Phys. J. C 72, 1896 (2012).

[52] M. L. Mangano, M. Moretti, F. Piccinini, and M. Treccani, J. High Energy Phys. 01 (2007) 013.

[53] F. Ambrogi, C. Arina, M. Backovic, J. Heisig, F. Maltoni, L. Mantani, O. Mattelaer, and G. Mohlabeng, Phys. Dark Universe 24, 100249 (2019).

[54] P. A. R. Ade et al. (Planck Collaboration), Astron. Astrophys. 594, A13 (2016).

[55] ATLAS Collaboration, CERN Report No. ATL-PHYSPUB-2019-033, 2019.

[56] A. M. Sirunyan et al. (CMS Collaboration), J. Instrum. 13, P10005 (2018).

[57] C. Chen, Phys. Rev. D 85, 034007 (2012).

[58] T. Chen and C. Guestrin, https://doi.org/10.1145/2939672 .2939785 (2016).

[59] T. Han, S. Mukhopadhyay, and X. Wang, Phys. Rev. D 98, 035026 (2018). 